PLASMA FIBRONECTIN IN PREMATURE NEONATES DURING EPISODE OF INFECTION AND CORRELATION WITH IMMUNOGLOBULIN ADMINISTRATION.

M.Anagnostakou, J.Peristeri, Th.Spanos, M.Christodouiou, N.Lipsou and M.Xanthou

$B^{-}$NICU "AGHIA SOPHIA" Children's Hospital, Goudi, Athens-Greece.

The aim of our study was to measure the concentrations of Fibronectin in LBW neonates during infections, as well as the correlation of blood levels with immunoglobul in administration. Fibronectin (FN) levels were measured from the serum of 16 premature neonates $B W=1150 \pm$ $260 \mathrm{gr}$ and $G A=29 \pm 2 \mathrm{w}$. Samples were taken weekly from the 30 th week of gestational age until the 38 th using the method of tholosimetrias. Ouring this period the neonates presented with 1 or 2 episodes of sepsis proved by clinical and laboratory investigation. Before or during infection, the neonates along with antibiotics, received sandoglobulin in total dose $2.5 \mathrm{~g}$ IV.Our results show a strong increase of FN levels between $30-34 w$ GA $p<0.007$, but we did not find a significant increase between $34-38 w$ p $<0.230$ in heal thy babies. Taking into consideration the babies with infection our results indicate that there was a severe drop in ibronectin levels. globulin had a less severe drop in Fibronect in levels than those who did not receive it $(p<0.038)$

Dutch Society of Psychosocial Consultation in Paediatrics (DSPCP)

(under the authority of the European Society of Child and Adolescent Psychiatry)

\title{
$\triangle 380$
}

Anti-epileptic therapy and behaviour disturbances in children Sergio Domizio, Patrizia Fontebasso, Alberto Verrotti, Giuseppe Sabatino

Department of Neonatology and Pediatric, University of Chieti,Italy Many studies have reported behavioral disturbances in children suffering from convulsive disorders, but there are conflicting data concerning the real incidence of this problem in the pediatric age group. In order to evaluate the effects on behaviour of some ant $i$ epileptic drugs, we studied 300 children treated with phenobarbital and other drugs; their age ranged from 3.1 months to 15.9 years. The children were divided into two groups: group A. 197 (116 male and 81 female) children female) children, mean age \pm D $5.3+2,8$ years, treated with phenobarbital; group B: 103 ( 66 male and 37 female) children, mean age $6.4 \pm 3.1$ years, treated with anti-epileptic drugs oth phenobarbital. In all patients hyperactivity, irritability, disturbances of sleep, and drowsiness were investigated. The parents of patients completed a questionnaire with seven items. In group A, $150(76.1 \%)$ children showed one or more behaviour disturbances, while in group B a smaller number of patients 32 (31\%) had such disorders. There was a significant difference between the two groups $(P<0.0001)$. The most frequent disorder was hyperactivity. In conclusion, the results of this study suggest that anti-epileptic drugs, in particular phenobarbital, can cause behaviour disturbances and we can underline the importance of careful evaluation of the risk/benefit ratio of these drugs.

\section{$\Delta 381$}

CONSTRUCTION OF ANKARA DEVELOPMENTAL SCREENING INVENTORY AND ITS ENVIRONMENTAL CORRELATES

Işık Savaşır "Hacettepe University", Nilhan Sezgin "Bilkent University", Neşe Erol "Ankara University", Ankara, TURKEY.

The aim of this study was to construct a culturally appropriate developmental inventory that would be useful in large scale surveys, assessing developmental changes over time both at individual and community level and evaluating progress of programs geared to the developmental needs. The impact of rearing environments on development was also investigated. The inventory was completed by 860 mothers of 0-6 year old children from three SES groups. Environmental variables were assessed by 2 other questionnaires. Results indicated that there were significant age and SES differences. As children grew older, their developmental scores on the inventory also increased. Significant SES differences were observed especially after the age of 3 years. There were no significant sex differences. The test-retest reliabilities and Cronbach alpha coefficients were high for the age groups. Our results indicate that some of the environmental variables had important impact on development.

\section{WITHDRAWN}

\section{$\Delta 383$}

PSYCHOSOCIAL FUNCTIONING IN JUVENILE CHRONIC ARTHRITIS Carol Fitzpatrick, Hugh Monaghan, Arn Deehan. Departments of Paediatrics and Child Psychiatry, Our Lady's Hospital for Sick Children, Cruml in, Dublin 12, Ireland.

The purpose of this study was to determine whether young people with
Juvenile Chronic Arthritis (JCA) differ from matched healthy controls on standardised measures of psychosocial functioning. The study group comprised 43 young people with JCA (age range $4-18$ yrs). The comprised 43 young people with JCA (age range 4 - 18 yrs). The
control group comprised 644 young people, who provided normative data control group comprised 644 young people, who provided normative data
for an Irish population. Assessment instruments included the child Behaviour Checklist (Achenbach \& Edelbrock) and the Youth Self Report for those aged 12 - 18 yrs.

Children (aged 4 - 11) with JCA scored significantly lower than heal thy controls on measures of competence, and significantly higher for internalizing problems, withdrawn behaviour, attention problems and total problems. Adolescents (aged 12 - 18) with JCA did not differ from healthy controls on the above measures, whether rated by the ir parents or by themselves.

Resuits suggest that age is an important factor in successful psychosocial functioning in JCA.

\section{$\triangle 384$}

"THE CHILD CONCEIVED TO GIVE LIFE: BONE MARROW TRANSPLLANTATION FROM PROGRAMATD DONOR, FIVE YEARS ON".

Roberto G. Burgio*B, Annapia Verri**B and Luigi Nespoli***

* Pediatrics Department, ** Neuropsychiatric Clinic, B University of Pavia, I-27100 - Pavia, Italy;

*** Pediatric Division, Ir Facoltà di Medicina e Chirurgia - I-21100 Varese, Italy.

Bone marrow transplantation (BMT) among children has been the subject of ethical and medico-legal problems. The usually juvenile condi tion of the do nor has frequently been one of the sources of these problems. Five years ago, we performed a BMT from a minor, programmed ghter with a compatible donor. A chronic myeloid Phl + leukemia was diagnosed two years before in the recipient, Evelina, and the BMT from a matched family donor was the only possible cure at that time. There have been stormy reactions among mass-media and mostly among the catholic community. The last expressed doubts on programming a child to secure the health of another child, like an inftrument. In our opinion, it is acceptable that parents program a child and know in advance whether he is compatible or not whereas it unethical to conceive a child to serve as a bone marrow donor and abort him if it conceive a child to serve as a bone marrow donor and abort him if it
is not compatible. There is no reason in considering a BMT from a "programmed" donor only as an utilitarian mean; rather after our expet rience we can assert that one life has been saved and another has be of the recipient and of the donor as well as of the parents has found 